\title{
The rewarding nature of provocation- focused rumination in women with borderline personality disorder: a preliminary fMRI investigation
}

\author{
Jessica R. Peters ${ }^{1 *}$, David S. Chester ${ }^{2}$, Erin C. Walsh ${ }^{3}$, C. Nathan DeWall ${ }^{4}$ and Ruth A. Baer ${ }^{4}$
}

\begin{abstract}
Background: Understanding why individuals with borderline personality disorder (BPD) ruminate on prior provocations, despite its negative outcomes, is crucial to improving interventions. Provocation-focused rumination may be rewarding in the short term by amplifying anger and producing feelings of justification, validation, and increased energy, while reducing self-directed negative affect. If provocation-focused rumination is utilized regularly as a rewarding emotion regulation strategy, it could result in increased activation in reward-related neural regions. The present pilot study examined neural correlates of provocation-focused rumination, relative to other forms of thought, in BPD.
\end{abstract}

Method: Functional magnetic resonance imaging (fMRI) was utilized to examine this theory in a pilot study of women diagnosed with BPD $(n=13)$ and healthy controls $(n=16)$. All participants received highly critical feedback on a previously written essay in the scanner, followed by prompts to engage in provocation-focused, self-focused, and neutral thought.

Results: Whole-brain analyses showed that in response to the provocation, participants with BPD (compared to controls) demonstrated increased activation in the ventrolateral prefrontal cortex (PFC). BPD participants also showed greater activation in the dorsomedial PFC during provocation-focused rumination (relative to neutral-focus). Subsequent $\mathrm{ROI}$ analyses revealed that provocation-focused rumination (compared to neutral-focus) increased activation in the nucleus accumbens for the BPD group only.

Conclusions: These findings, while preliminary due to the small sample size and limitations of the protocol, provide initial data consistent with the proposed neurobiological mechanism promoting provocation-focused rumination in BPD. Directions for further research are discussed.

Keywords: Borderline personality disorder, Anger, Rumination, Reward, fMRl, Criticism, Nucleus accumbens, Insula, Provocation

\section{Background}

Borderline personality disorder (BPD) is characterized by affective instability, identity disturbances, problems in interpersonal relationships, intense anger, and self-destructive impulsivity [1]. Rumination, defined as repetitive, passive, unconstructive thinking about negative emotions and problems [2], may contribute to amplifying and maintaining

\footnotetext{
* Correspondence: jrpeters@gmail.com

${ }^{1}$ Department of Psychiatry and Human Behavior, Alpert Medical School of Brown University and Rhode Island Hospital, 700 Butler Drive, Providence, R 02906, USA

Full list of author information is available at the end of the article
}

these patterns of negative affect and dysfunctional behavior. Although many people falsely assume that extended thinking about problems will lead to insight and solutions [3, 4], rumination intensifies negative affect and reduces problemsolving ability. Anger rumination, in which individuals focus on angry moods and prior provocations, is particularly associated with BPD features $[5,6]$ and predicts characteristics of BPD, such as anger, displaced aggression, and cognitive distortions [7-9]. While individuals with BPD vary in the extent to which they anger ruminate, these robust findings suggest it is a common behavior in this population. To develop more effective treatments, it is 
crucial to understand why individuals with BPD tend to engage in rumination on provocations and anger despite its negative outcomes.

\section{Function of anger rumination in BPD}

One proposed function of anger rumination is avoidance of more aversive emotions and cognitions [10]. Shame proneness is endemic to BPD [11-13], as is pronounced rejection sensitivity [14-16]. Ruminating on anger may reduce this internally directed negative affect, by focusing instead on external causes for distress, such as unfair situations and deplorable behavior of others [13]. The resulting amplified anger contributes to the aggression and interpersonal problems typical of BPD, potentially increasing risk of future social rejection and feelings of shame. Consistent with this theory, self-reported anger rumination and anger have been shown to mediate the relationship between shame-proneness and BPD features in a student sample [13], and individuals diagnosed with BPD have been shown to react strongly to rejection cues with rage [17].

Anger is typically conceptualized as a negative emotion, but it also has immediate positive outcomes, such as increased energy and feelings of justification. Most negative emotions induce avoidant behavior; however, like positive affect, anger increases approach motivation [18]. Therefore, anger rumination may not only dampen BPD individuals' self-directed negative affect (negative reinforcement), but also provide feelings of validation, empowerment, and pleasure (positive reinforcement). These positive immediate effects may be particularly reinforcing given that interpersonal experiences typically considered rewarding, such as praise from others, fail to produce positive affect for individuals with BPD and may actually be evaluated negatively [19]. If the theory proposed is accurate, individuals with BPD should experience altered neural activity in reward networks during provocation-focused rumination, following increased neural reactivity to critical feedback.

\section{Neural correlates of reactivity to criticism}

In healthy individuals, social exclusion activates neural regions involved in affective distress, including the dorsal anterior cingulate cortex (dACC) and the anterior insula (AI), as well as the ventrolateral prefrontal cortex (VLPFC) $[20,21]$. Activation of this system may function as a neural alarm that promotes recognition of and responses to the event [21]. Alterations in these neural regions have been implicated in reactivity to rejection and interpersonal interactions in BPD. In one study comparing neural reactivity to rejection, inclusion, and neutral conditions during a behavioral task in individuals with BPD and healthy controls, the rejection condition elicited relatively greater dACC activation compared to inclusion and neutral conditions within each group as expected; however, the BPD group also demonstrated a main effect of higher levels of dACC and dorsomedial prefrontal cortex (DMPFC) activation relative to controls across all task conditions [22]. These findings suggest that, while activation in the dACC may increase in response to rejection for both individuals with BPD and controls, for those with BPD, this may occur in conjunction with a generally heightened level of activation in this system when evaluating social situations.

The VLPFC, which co-activates with the dACC and insula in response to social exclusion, is associated with regulation of negative emotions and inhibition of psychological pain $[20,23,24]$. Amplifying activation in the VLPFC prior to and during a social exclusion paradigm attenuated emotional reactivity [25]. Conversely, inhibiting the VLPFC following social exclusion amplified the normative negative emotional response [26]. Together, these findings suggest a key role for the VLPFC in regulating affective reactivity to social rejection.

\section{Neural correlates of anger rumination}

Several neural regions have been specifically linked to anger rumination in a non-clinical sample. Denson et al. [27] employed an interpersonal provocation manipulation where an experimenter was rude and implied participants were not intelligent enough to follow directions. Then, during fMRI scanning, the participants received sets of prompts (counter-balanced in order) to engage in various forms of thought: provocation-focused (e.g., "Think about how you have interacted with the experimenter up to this point"), self-focused (e.g., "Think about why you react the way you do"), and neutral-focused (e.g., "Think about the layout of the local post-office") [27]. Compared to neutralfocus, both provocation- and self-focused conditions involved greater recruitment of regions related to anger and affective responses to social rejection (dACC), emotion regulation (LPFC), arousal (thalamus, insula), and self-referential thought (dorsomedial prefrontal cortex; DMPFC). Activation of the DMPFC and right anterior insula across both rumination conditions, compared to the neutral-focused condition, correlated with self-reported state rumination and trait-level displaced aggression. The study did not obtain any whole-brain findings for rewardrelated regions in this non-clinical sample, nor were any reward-related ROIs hypothesized about or examined.

\section{Neural correlates of reward}

Positive reinforcement activates the ventral striatum, specifically the nucleus accumbens (NAcc), a central node in learning, motivation, and reward circuitry see [28] for review. Recruitment of the NAcc has most reliably been associated with experiences of reward and subjective pleasure [29-31], occurring in response to a range of appetitive cues and pleasurable activities including both naturally occurring 
rewards (e.g., money, food, orgasm) and drugs of abuse [32]. Additionally, pleasant mental imagery also been selectively activates the NAcc and the MPFC, with the degree of NAcc activation correlated with the extent of pleasure endorsed [33].

While individuals with addiction behaviors, such as substance abuse, tend to demonstrate baseline hypoactivity of reward networks, these regions, including the NAcc, show increased activation during anticipation of relevant appetitive cues [34]. This NAcc sensitization to rewarding stimuli creates a learned motivational response facilitating addiction even in the absence of withdrawal symptoms [35], suggesting this process could also facilitate nondrug habits. Consistent with this, NAcc sensitization has been demonstrated during the anticipation of eating [36], planning of food binges [37], and decision-making about retaliatory aggressive behavior [38] for individuals with maladaptive levels of these behaviors in daily life.

BPD-specific striatal alterations may be linked to difficulties with emotion regulation. Striatal regions functioned similarly in BPD patients and controls in response to monetary rewards in emotionally neutral contexts; however, in the context of emotional images, BPD patients demonstrated reduced reward differentiation and less deactivation of reward circuitry following cue exposure [39]. One possibility is that emotional reactivity disrupts reward systems for BPD patients [39]. Alternatively, for emotionally reactive individuals, emotional cues could have greater potency as reward or punishment than small amounts of money. These findings hint at the possibility that emotionally evocative stimuli and processes, such as anger rumination, may function as BPD-relevant appetitive cues.

\section{Clarifying the function of provocation-focused rumination in BPD}

The present pilot study utilized fMRI to compare blood oxygen level dependent (BOLD) signal changes in specific brain regions among participants with BPD and healthy controls across the experiences of interpersonal provocation and ruminative responding. In response to provocation, participants with BPD (vs. controls) were expected to demonstrate higher activation in brain regions associated with reactivity to social rejection (AI, dACC, VLPFC). All participants were expected to demonstrate greater activation in regions previously associated with anger rumination (dACC, DMPFC) during subsequent provocation-focused thought compared to neutral-focused thought; however, this effect was expected to be greater for participants with BPD. Participants with BPD (vs. controls) were predicted to experience greater activation in brain regions associated with reward and pleasure (NAcc) during provocation-focused thought.

\section{Methods}

\section{Participants}

Participants $(n=31)$ were right-handed women who were at least 18 years old. Thirteen of them met the DSM-IV criteria for BPD. The other 18 were age-matched healthy controls. All participants were screened for suitability for MRI research. Individuals were excluded who reported neurological pathology or injury, developmental disorders, prior or current problematic substance use, psychotic symptoms, and claustrophobia (determined through interview with the participant about their lifetime history of diagnoses, injuries, substance use, and discomfort in enclosed spaces, as well as several questions assessing delusions and hallucinations) - these screeners were conducted on the phone and then repeated in-person. Control participants were required to meet no criteria for BPD and to have never received any other psychological diagnosis or treatment and not to be using psychoactive medication. Of the BPD group, 11 were not on any psychoactive substances at the time of the study, and 2 were taking SSRI medication. Only one member of the BPD group had recently begun receiving dialectical behavior therapy; most other BPD group participants reported prior lifetime experience with psychotherapy (not BPD-specific), however were not currently in therapy for a range of reasons (e.g., previous therapy was not helpful, finances). All participants were offered low-cost psychotherapy referral options following the experiment as part of the debriefing process.

Recruitment occurred from contacts with local clinics and psychotherapists, craigslist advertisements, study fliers, and introductory psychology classes at a large, public university. Participants received either $\$ 100$ for participating or course credit. Advertisements for the BPD group did not mention BPD specifically, given that prior BPD diagnosis was not required; instead, the fliers read, "You may be eligible to participate if experience intense emotions and difficulties in relationships." Participants who responded to the advertisement and expressed interest were then administered a phone screener for BPD symptoms. For the BPD group, those who endorsed 5 or more criteria on a brief phone screen based on the complete BPD diagnostic interview $(N=22)$ were invited to participate; only those who met criteria for BPD during the in-person diagnostic interview $(N=17)$ were asked to return for the scanning session. Of those, 14 returned for the scan session. Two of the final BPD group participants were recruited from the psychology classes, one from an outpatient clinic, and eleven from the general community.

\section{Measures}

Structured clinical interview for the DSM-IV Axis II disorders (SCID-II; [40])

The SCID-II is a standardized, semi-structured, clinician administered interview for diagnosing DSM-IV Axis II 
mental disorders. The BPD section only of the SCID-II was administered by an advanced doctoral candidate in clinical psychology and interviews and scoring reviewed with a licensed clinical psychologist.

\section{Personality assessment inventory borderline features scale (PAI-BOR; [41])}

The PAI-BOR is a well-validated measure of four aspects of BPD pathology: affective instability, identity problems, negative relationships, and self-harm. Raw scores on the total scale above $37(T>70)$ are considered to be in the clinical range and predict BPD-specific dysfunction in clinical, community, and student samples [41, 42], while raw scores below $18(T<30)$ represent absent to minimal BPD-related symptoms. In the present study, PAI-BOR total score demonstrated good to excellent internal consistency $(\alpha=.84-.96)$.

\section{Anger rumination scale (ARS; [43])}

The ARS has 19 items assessing the tendency to focus attention on angry moods, recall past anger episodes, and think about the causes and consequence of anger episodes. Responses range from 1 ("almost never") to 4 ("almost always"). The ARS total score demonstrated excellent internal consistency in the present study $(\alpha=.96)$.

\section{Center for Epidemiological Studies_Depression (CES-D; [44])}

The CES-D is a 20-item inventory of depressive symptoms. The CES-D asks participants to rate their mood, thoughts, and behavior during the previous week on a 4-point Likert scale, ranging from 0 ("rarely or none of the time") to 3 ("most or all of the time"). In the present study, the CES-D demonstrated excellent internal consistency $(\alpha=.94)$.

\section{PTSD checklist-Civilian version (PCL-C; [45])}

The PCL-C is a 17-item questionnaire that asks participants to rate the extent they have been bothered by PTSD symptoms over the past month. Responses range from 1 ("not at all") to 5 ("extremely"). In the present study, the PCL demonstrated excellent internal consistency $(\alpha=.95)$.

\section{Procedure}

\section{Preliminary screening}

A phone screen was administered to all potential participants including the diagnostic and MRI safety screeners. Participants were also administered a risk assessment; this was repeated in person for individuals who enrolled in the study and to ensure safety at points throughout the study. Individuals were excluded for current urges to engage in harm to self or others; participants in the BPD group could endorse lifetime self-harm or suicidality. These phone interviews and all subsequent clinical interviews and assessments were conducted by an advanced clinical psychology doctoral student.

\section{Assessment session}

Participants $(N=43)$ completed self-report measures of BPD symptoms, and the SCID-II for BPD was then administered. Any participants who did not meet inclusion criteria (no BPD criteria met for the control group; at least five BPD criteria fully endorsed for the BPD group) were excluded from the second study session.

\section{Scanning session}

Participants $(N=31)$ completed the scanning session, which took place between 2 and 10 days after the assessment visit.

Essay-writing paradigm Participants were asked to write a short essay about a time in which someone else angered them. In accordance with a previously validated provocation paradigm [46], they were told that a research assistant would evaluate it on several key criteria and that this feedback would be provided while they are in the MRI scanner. Each participant's essay was given the same harsh criticism, regardless of what they had written (see Scanning Procedure).

Scanning procedure Each MRI scanning session included two experimental tasks that were completed while fMRI was acquired. After a high-resolution anatomical scan was completed, participants were removed from the scanner.

Provocation Task. The provocation manipulation was created by combining a previously-used fMRI provocation procedure [27] with an insulting essay feedback paradigm used in behavioral research, that has previously been demonstrated to produce a robust increase in anger [46]. This procedure was chosen over the one previously used with directed rumination task (where the experimenter personally delivered the provocation), in order to maintain the alliance between the experimenter and participant in the event of safety concerns, given the clinical sample. The task was divided into three blocks (pre-feedback baseline, feedback, post-feedback baseline) [27]. In the first, pre-feedback block, participants passively viewed a fixation cross to capture baseline neural activity (120 s). Next, participants viewed a prompt to "Get Ready to View Your Essay Feedback" (5 s). Then, participants viewed a series of five ratings of various characteristics of their essay (10 s per rating; e.g., "clarity of expression", "writing style") that were preprogrammed to be insulting (1/7-3/7 points) as well as a total score $(10 / 35 ; 10 \mathrm{~s})$. All participants received the same ratings. Then, participants viewed their reviewer's 'comments' on their essay for $10 \mathrm{~s}$, which was: "One of the worst essays I have EVER read!" Finally, participants viewed another fixation cross to model post-feedback baseline neural activity (120 s). 
Directed Rumination Task (DRT). To assess neural activity specific to angry rumination (i.e., repetitive thoughts about the provocation participants had just experienced), participants completed a shortened version of a previously validated paradigm in which participants are directed to ruminate about three topics in succession: the prior provocation (provocation-focus condition), themselves (self-focused condition), and a neutral topic (neutralfocus condition) [27]. The three block-types were presented in counter-balanced order across participants, within groups. The task was implemented in a block design. In each block, participants viewed a series of 6 statements (15 s per statement; 90s per block), that instructed participants what content to ruminate about. ${ }^{1}$ During provocation-focused blocks, participants read rumination prompts with statements instructing them to engage in anger rumination, reflecting on the provoking incident encountered earlier in the study (e.g., "Think about how you have been treated" "Think about whether your treatment was unfair or unreasonable"). During selffocused blocks, participants read statements instructing individuals to think about themselves (e.g., "Think about what kind of a person you are." "Think about why you respond to others the way you do."). During neutralfocused blocks, participants read prompts with statements instructing individuals to reflect on neutral statements unrelated to the study (e.g., "Think about the layout of the local post office", 'Think about a bus driving down the street"). Between blocks of the DRT, participants were given a $30 \mathrm{~s}$ rest period with a fixation cross, followed by a 5 -s prompt to get ready for the next set of statements.

After exiting the scanner, participants were told of the deception involved in the writing task and provocation.

\section{Data acquisition and analyses fMRI data acquisition}

All images were collected on a 3.0 T Siemens Magnetom Trio scanner using a Siemens 32-channel head coil. Functional echo planar images were acquired with a T2*-weighted gradient echo sequence with a 3D shim applied before functional data acquisition (matrix size $=$ $64 \times 64$, field of view $=224 \mathrm{~mm}$, echo time $=28 \mathrm{~ms}$, repetition time $=2.5 \mathrm{~s}$, slice thickness $=3.5 \mathrm{~mm}, 40$ interleaved axial slices, flip angle $=90^{\circ}$ ). These parameters allowed for whole-brain coverage with $3.5 \mathrm{~mm}$ cubic voxels. A high-resolution, coplanar T1-weighted image was also acquired from each participant so that functional data could be registered to native anatomical space and then normalized to the Montreal Neurological Institute $(\mathrm{MNI})$ atlas space $\left(1 \mathrm{~mm}^{3}\right.$ isotropic voxel size, echo time $=2.56 \mathrm{~ms}$, repetition time $=1.69 \mathrm{~s}$, flip angle $=12^{\circ}$ ).

\section{fMRI preprocessing}

All preprocessing and statistical analyses were conducted using FSL (Oxford Center for Functional Magnetic Resonance Imaging [FMRIB] $[47,48]$ ). Functional volumes were reconstructed from k-space and the reconstructed functional volumes were corrected for head movement to the median volume using MCFLIRT [49], corrected for interleaved slice-timing skew using temporal sync interpolation, pre-whitened using FILM, and spatially-smoothed with a $5 \mathrm{~mm}$ full-width-half-maximum Gaussian kernel. To remove drifts within sessions, a high-pass filter was applied (200 s cutoff). Non-brain structures were stripped from functional and anatomical volumes using FSL's Brain Extraction Tool [50].

\section{fMRI data analyses}

We modeled within-subjects, between-subjects and between-groups (BPD vs. control) variance in brain activation utilizing a 2-stage summary statistics approach to multi-level modeling via FSL. An initial fixed-effects general linear model (GLM) modeled event-related responses for each run of each participant using a canonical double-gamma hemodynamic response function with a temporal derivative. All six motion parameters were modeled as nuisance regressors for all analyses. For the Provocation Task, pre-feedback baseline, feedback, and post-feedback baseline blocks were each separately modeled as regressors in the model, with pre-block instructions modeled as a nuisance regressor. Within the Provocation Task, we contrasted feedback with pre-feedback baseline (feedback > pre-feedback baseline), to assess the effects of critical feedback on activation. For the DRT, provocationfocus, self-focus, and neutral-focus blocks were modeled as regressors in the first-level GLM. Pre-block instructions were modeled as a nuisance regressor and fixation trials were left unmodeled to serve as an implicit baseline. Within the DRT task, we separately contrasted provocation-focus with both self-focus and neutral-focus blocks, as well as self-focus contrasted with neutral-focus, to assess activation specific to each of those conditions.

Whole-brain analysis To model these contrasts at the group level, we performed top-level, mixed-effects GLM analyses, which created group average maps for contrasts of interest and allowed us to contrast BPD and control groups. For each lower-level contrast (e.g., provocationfocused rumination $>$ self-focused rumination), grouplevel $Z$ (Gaussianized T/F) statistic images were created and then thresholded using clusters determined by $Z>2.3$ and a (familywise error corrected) cluster significance threshold of $p<.05$. In addition to these group-level aggregate analyses, we created contrast maps that compared BPD participants to controls using the same thresholding 
procedures previously described. Cluster thresholding was applied both across the whole brain.

Regions of interest analysis We also employed an a priori region of interest (ROI) approach for the DRT task to investigate the effects of rumination on ROIs implicated in anger rumination and reward processing. Four ROIs in the DMPFC (left superior DMPFC, right superior DMPFC, left medial DMPFC, and right medial DMPFC) and two in the dACC (right dACC, left dACC) were based on an activation clusters found in previous research on activation in these regions during anger rumination, compared to neural thought [27]. Each ROI was constructed using an $8 \mathrm{~mm}$-radius sphere around each cluster's peak voxel. Given that no published studies to date have directly examined the effects of provocationrelated rumination on reward-related brain regions, we also examined ROIs in order to provide critical preliminary tests of our central hypothesis. Region of interest (ROI) masks were constructed for the right and left NAcc from the Wake Forest Pickatlas toolkit [51]. For each task condition, parameter estimates were extracted (in units of percent signal change) and averaged across all voxels of each ROI. Parameter estimates were then analyzed in SPSS via Group (BPD, Control) x Condition (Provocation-, Self-, NeutralFocus) ANOVA, with Bonferroni corrections employed for post-hoc contrasts to control for familywise error rates.

\section{Power}

Power was estimated for comparisons across the DRT, the primary analyses of interest. For between and withinsubject effects in the GLM, power ranged from 11 to $14 \%$ for small effects $(d=.2), 42-66 \%$ for medium effects $(d=.5)$, and $80-98 \%$ for large effects $(d=.8)$, based on Cohen's effect sizes [52]. The study was a relatively small, preliminary exploration of a novel theory; accordingly, it was not powered to detect smaller effects.

\section{Results}

\section{Data screening}

Data were screened for outliers on all measures. One participant was removed from analyses due to values greater than $3 \mathrm{SD}$ above the mean for the entire sample for activation of the right and bilateral NAcc during the provocation $>$ neutral contrast during the DRT. One control participant was removed prior to analyses due to endorsement of one of the DSM BPD criteria during the debriefing session. The final sample analyzed included 28 participants $($ BPD group $=13$; control group $=16$ ).

\section{Demographics and self-report}

Groups did not significantly differ by age (see Table 1 ), race $\left(\chi^{2}=.76, p=.69\right)$, or education level $\left(x^{2}=4.12\right.$, $p=.13)$. Accordingly, these demographic variables
Table 1 Differences between control and BPD groups on selfreport measures of BPD symptoms, anger rumination, and age $(N=29)$

\begin{tabular}{lllll}
\hline & HC Mean (SD) & BPD Mean (SD) & $\mathrm{t}$ & $p$-value \\
\hline PAI-BOR & $9.88(5.02)$ & $44.23(8.75)$ & 13.28 & $<.001$ \\
ARS & $1.26(.20)$ & $2.55(.37)$ & $11.32^{\mathrm{a}}$ & $<.001$ \\
CES-D & $7.44(6.65)$ & $28.23(9.27)$ & 7.03 & $<.001$ \\
PCL & $23.69(8.65)$ & $49.31(13.21)$ & 6.29 & $<.001$ \\
Age & $21.81(4.02)$ & $21.23(3.30)$ & -.42 & .58 \\
\hline
\end{tabular}

PAI-BOR Personality Assessment Inventory-Borderline Features Subscale, ARS Anger Rumination Scale, CES-D Center for Epidemiological Studies Depression Scale, PCL Post-Traumatic Stress Disorder Checklist

$t$-tests conducted with equal variances assumed except where denoted by $\left({ }^{a}\right)$

were not controlled for in subsequent analyses. To confirm validity of SCID-II diagnoses, PAI-BOR scores for the BPD group were compared to the control group (see Table 1 for group comparisons of all self-report variables). As expected, the BPD group reported significantly higher levels of BPD symptoms. The control group endorsed a mean level of PAI-BOR total scores in the low symptoms category, with no control participants reporting above average symptom levels, whereas the BPD group's mean was clinically elevated, with $85 \%$ reporting clinically elevated symptoms and two participants endorsing above average levels. Also consistent with previous studies, the BPD group reported generally engaging in a significantly higher level of anger rumination than the control group. The BPD group also reported a significantly greater level of symptoms of depression and PTSD than the controls. Group means for the CES-D were similar to previous studies comparing women with BPD to healthy controls [11], with $1(6 \%)$ control participant and 10 (77\%) BPD participants endorsing symptom levels consistent with elevated risk of depression. For the PCL-C, 1 (6\%) control participant and 8 (62\%) BPD participants endorsed symptom levels above the screening thresholds for elevated risk for PTSD.

\section{Imaging results \\ Provocation task}

In the whole-brain analyses, the BPD > Control betweengroup contrast revealed a cluster of increased activation in the VLPFC (peak coordinates: inferior frontal gyrus as defined by Harvard-Oxford cortical structural probabilistic atlas) that extends into the orbitofrontal cortex, operculum, and anterior insula, in response to the negative essay feedback (Fig. 1, feedback > pre-feedback baseline contrast; Table 2).

\section{Directed rumination task}

Whole-brain analysis Whole-brain analyses revealed a significant between-group difference in neural activation 


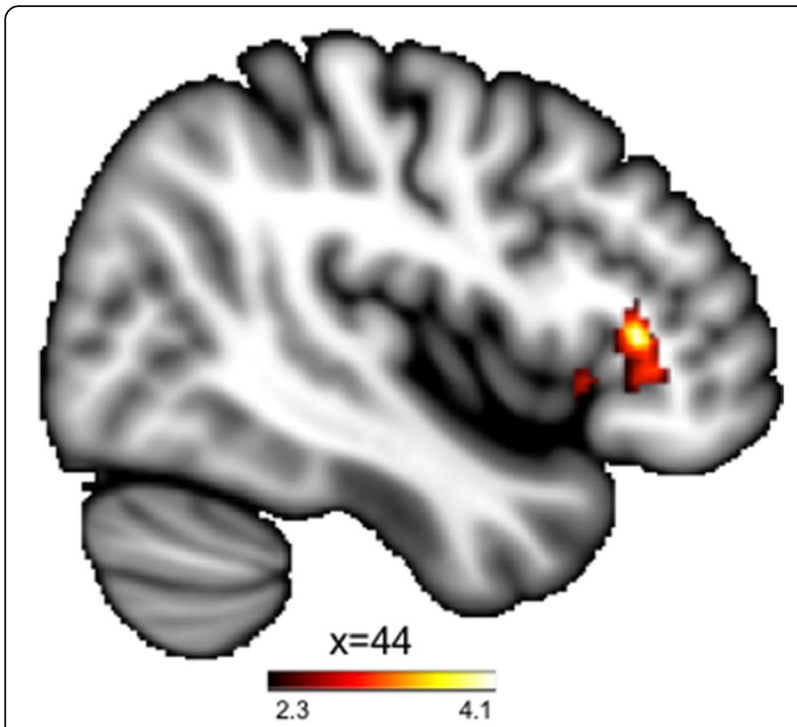

Fig. 1 During the Provocation Task, greater activation of the right VLPFC was observed among BPD participants (compared to Controls)

on the Directed Rumination Task. The BPD $>$ Control contrast revealed greater activation in midline DMPFC during provocation-focused rumination (as compared to the neutral condition; Fig. 2; Table 2). No significant effects of BPD diagnosis were found on self-focused rumination (as compared to the neutral condition).

ROI analysis To reduce familywise error, the DMPFC ROIs were combined into a single index averaging across the ROIs, demonstrating high internal consistency within each DRT contrast $(\alpha>.88)$. The DACC ROIs were similarly combined into a single index for each contrast $(\alpha>$.93). Right and left NAcc ROIs were also combined into an index representing bilateral NAcc activation for each contrast $(\alpha>.76){ }^{2}$

GLM analyses demonstrated no group by condition interaction on DMPFC activation (F $[2,54]=1.47, p=.24)$, but a significant main effect of DRT condition on this DMPFC index (F $[2,54]=4.96, p=.010, d=.86)$. Posthoc contrasts revealed significant greater activation in the provocation condition compared to neutral $(t=$ $4.10, p<.001, d=.76)$ and the self-condition compared to neutral $(t=2.73, p=.011, d=.50)$, with no significant

Table 2 Regions of activation in the BPD group vs controls

\begin{tabular}{llll}
\hline Region & MNl coordinates & $k$ & Peak z-score \\
\cline { 2 - 3 } & X Y & & \\
\hline
\end{tabular}

Critical Essay Feedback: Provocation paradigm > Baseline

$\begin{array}{llllll}\text { Right VLPFC } & 44 & 30 & 6 & 397 & 4.14\end{array}$

Directed Rumination Task: Provocation-focused thought $>$ Neutralfocused thought

DMPFC

2

48

38

696

4.46

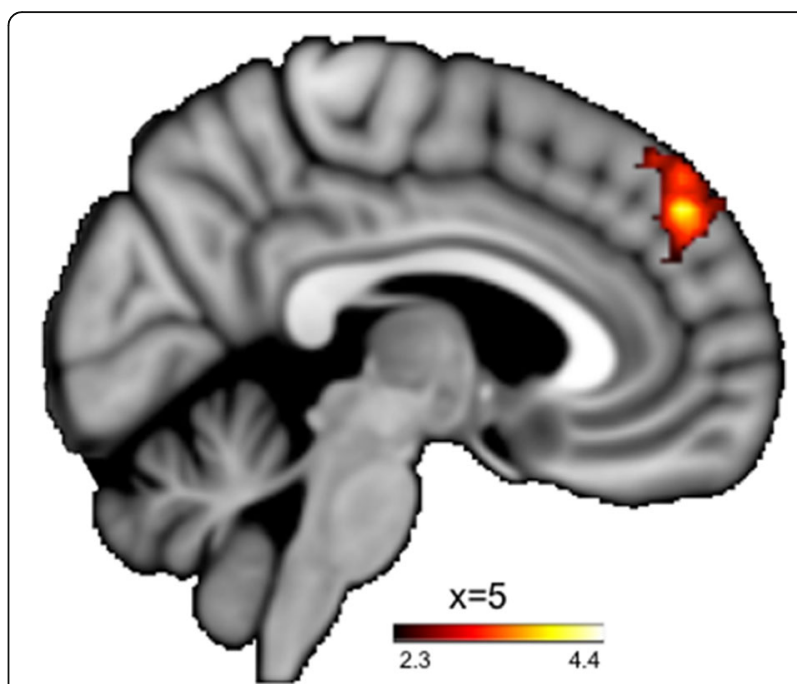

Fig. 2 During the Directed Rumination Task, BPD participants (compared to Controls) showed increased activation of the DMPFC provocation-focus (vs. neutral-focus)

contrast between the provocation and self-focused condition $(t=.82, p=.42)$. A similar pattern of findings emerged for the dACC ROI, with no group by condition effects $(\mathrm{F}[2,54]=.32, p=.73)$, but a significant main effect of DRT condition (F $[2,54]=3.86, p=.027, d=.76$ ), with post-hoc testing revealing significantly greater activation in the provocation-focused compared to neutral condition $(t=3.05, p=.005, d=.57)$, with no significant contrasts for the self-focused condition $(p>.22)$.

Second, we examined bilateral NAcc activation across the conditions of the DRT. GLMs estimating NAcc activation from condition, group, and condition by group interaction were modeled. When all three DRT conditions were included in the models, neither a significant group by condition interaction effect on the bilateral NAcc was observed $(\mathrm{F}[2,54]=2.56, p=.087)$ nor a main effect of condition alone was observed $(\mathrm{F}[2,54]=$ $2.19, p=.12$ ).

Given the DMPFC and dACC ROI findings suggesting that the self-focused condition was not well differentiated from the provocation condition, exploratory GLMs for the NAcc were estimated containing only the provocationfocused and neutral-focused conditions, to test the contrast of primary interest. A significant group by condition interaction, with a large effect size, was found for NAcc right activation $(\mathrm{F}[1,27]=6.38, p=.018, d=0.94)$. Probing this interaction demonstrates that, as hypothesized, for individuals with BPD, the provocation-focus condition, compared to neutral focus, led to increased activation in the NAcc $(\mathrm{t}$ $[12]=2.27, p=.018, d=0.76$ ), whereas for controls, no significant differences between these two conditions were observed ( $\mathrm{t}[15]=-.41, p=.69$; see Fig. 3 for DRT condition contrasts in bilateral NAcc activation by group). 


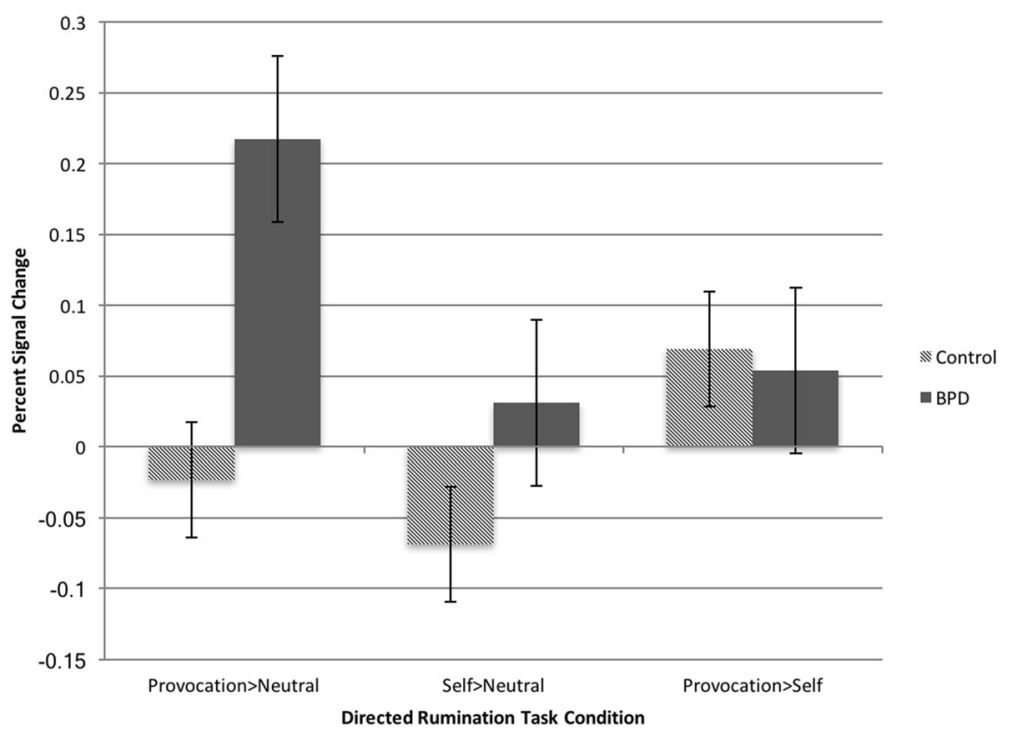

Fig. 3 Nucleus accumbens percent signal change for task contrasts by diagnostic group (BPD vs Control) during the Directed Rumination Task

As a post-hoc exploratory analysis, associations between right NAcc activation contrasts and self-reported BPD features, anger rumination, depression symptoms, and PTSD symptoms were also computed, using Spearman-Rank correlations given non-normal distribution of variables (see Table 3). The provocation $>$ neutral contrast demonstrated significant, moderate associations with the PAI-BOR features of self-harm, negative relationships, and affective instability. Associations with self-reported anger rumination, depression, PTSD symptoms, and identity disturbance were not significant, although effect sizes observed were moderate across all variables. No significant associations were observed between the self $>$ neutral contrast nor the provocation > self contrast and any of the self-report variables. Intercorrelations between all self-report measures were generally extremely high $(r \mathrm{~s}=.75-.86)$, with associations with the PAI-BOR self-harm subscale and others slightly lower $(r=.64-72)$.

\section{Discussion}

The results of this pilot study demonstrated mixed support for our hypotheses; however, they do provide some preliminary data consistent with the hypothesis that provocation- focused rumination may selectively activate neural regions associated with reward for individuals with BPD. While engaging in provocation-focused thought, relative to neutralfocus, all participants demonstrated greater activation in most of the regions previously associated with anger rumination and self-referential thought (DMPFC, dACC) [27], suggesting both groups engaged in the task; however, greater relative activation in regions of the DMPFC in provocation-focused thought in individuals with BPD, compared to controls, perhaps reflects greater intensity of engagement with the provocation stimuli for the BPD group.

ROI analyses showed differences in neural activation in regions associated with reward responsiveness during the DRT. Although the hypothesized group by condition interaction across all three directed rumination task conditions was not significant for NAcc activation, exploratory contrasts between the provocation- and neutral-focused conditions only revealed that while controls demonstrated no differences in reward-activation (NAcc ROI activation) between the neutral-focused and provocation-focused conditions, the provocation-focused condition produced significantly more activation in the right NAcc than neutral-focused thought for the BPD group. Furthermore,

Table 3 Spearman-rank correlations between bilateral nucleus accumbens activation contrasts during the Directed Rumination Task and self-report measures of BPD features, anger rumination, and symptoms of depression and PTSD $(N=29)$

\begin{tabular}{|c|c|c|c|c|c|c|c|}
\hline Condition contrast & ARS & PAI-BOR Al & PAI-BOR ID & PAI-BOR NR & PAI-BOR SH & CES-D & $\overline{\mathrm{PCL}}$ \\
\hline Provocation > Neutral & .31 & $.37^{\mathrm{a}}$ & .33 & $.46^{\mathrm{a}}$ & $.46^{\mathrm{a}}$ & .37 & .32 \\
\hline Self $>$ Neutral & .24 & .18 & .31 & .25 & .18 & .34 & .22 \\
\hline Provocation > Self & .06 & .13 & -.02 & .14 & .21 & .01 & .07 \\
\hline
\end{tabular}

${ }^{\mathrm{a}} p<.05$

ARS Anger Rumination Scale, PAI-BOR Personality Assessment Inventory Borderline Personality Disorder Scale, AI Affective Instability, ID Identity Disturbance, NR Negative Relationships, SH Self-Harm, CES-D Center for Epidemiological Studies Depression Scale, PCL Post-Traumatic Stress Disorder Checklist 
this increased NAcc activation during provocation-focused vs neutral-thought was correlated across the sample with self-report measures of BPD features of affective instability, negative relationships and self-destructive behavior.

Combined with the finding of greater activation in the right VLPFC and parts of the AI for the BPD group during the prior critical feedback, these findings are consistent with the theory that individuals with BPD are more reactive to criticism, possibly including greater efforts to regulate their emotional responses, and that they may find the experience of ruminating about the provocation more rewarding than healthy controls. This sequence of reactions could contribute to the well-established tendency of individuals with BPD to endorse high trait levels of both internally directed negative affect (shame) and externally directed negative affect (anger), aggression, and impulsive behavior. However, this interpretation of the data is limited by its use of reverse inference, and it is possible that these patterns of neural activation may reflect different or additional neural processes. For example, activation of the ventral striatum (including the NAcc) could reflect emotional enhancement of learning [53]. Further work combining neuroimaging with additional, task-based methods to measure emotional reactivity and reward responses would provide more robust tests of this theory that rely less on reverse inference. The interpretation is also limited by the lack of affect ratings or other non-neural data of participant reactivity to the inductions; without these, it is difficult to know whether there were group differences in affect, attribution, or interpretation of the task and precisely what form of affect was elicited during criticism and the rumination prompts and how these may have varied across participants or groups. The present analyses utilized ROIs previously linked to anger rumination and anger in the study that developed this task; however, it cannot be confirmed that these ROIs are linked to the same subjective experiences in the present sample. Further research is needed to establish whether these findings are specific to rumination following increased subjective experiences of anger; if confirmed, that would provide a stronger and more specific link between NAcc activation during anger-related rumination in BPD and better support for the theory in question.

As hypothesized, the BPD group demonstrated greater recruitment of the VLPFC, as well portions of the AI, when receiving critical feedback than controls. Activation of the VLPFC occurs in emotion regulation efforts, including those that result in increased negative affect [23]. The peak area of greater activation for the BPD group was in the inferior frontal gyrus, a region that may play a key role in efforts to engage in response inhibition [54]. Its activation may represent detection of a salient response regardless of eventual behavioral action [54]. Findings of greater reactivity in parts of the $\mathrm{AI}$ also are consistent with previous work on reactivity to distress in BPD, with a meta- analysis of negative emotion processing concluding that patients with BPD demonstrate hyper-reactivity in the right insular cortex [55]. Thus, in the present study, these findings may represent greater salience of the criticism, greater perception of the criticism as distressing, and/or greater effort required by the BPD group to process and attempt to regulate their emotional responses to the criticism.

Contrary to hypotheses, there were no significant differences between groups in $\mathrm{dACC}$ activation during this task. Some previous findings demonstrate deactivation of the ACC in BPD in negative emotion inductions, and the present results are consistent with a theory that the strong response in the insula to distress may, for some individuals with BPD, lead to suppression of ACC activation and thus facilitate dissociative experiences [56]. Another issue to consider is that individuals with BPD may be more reactive to stimuli conforming to BPD-specific themes (e.g., rejection and abandonment) $[57,58]$. The critical feedback may have been experienced as both notice of having done poorly on the task and also as potentially unfair, but not as an incident of social rejection. Different effects in both the feedback phase and following ruminative thought might be achieved if a more explicitly interpersonal critique had been levied, such as critical feedback regarding the person's potential as a friend after meeting them. Future work should incorporate these to determine more precisely the nature of the group differences in responses to the manipulations. A limitation of these findings is that the provocation > baseline contrast includes both provocation and other processes (including reading, social cognition, and selfevaluation); future studies should examine these effects using an active baseline control involving similar processes, such as reading neutral evaluations.

Similar to previous research [27], in the ROI analyses, the self-focus condition did not produce significantly different levels of NAcc or DACC activation from the other conditions for either group, and did not differ from the provocation condition in DMPFC activation. For nonclinical individuals, none of these forms of thought differentially activated the NAcc, whereas for individuals with BPD, focusing on the self may fall at an indistinguishable midpoint between neutral-focused and provocationfocused. One possible explanation for this finding is that the self-focused prompts may also invoke components of anger, particularly following an angering experience for the BPD group. Future research utilizing other more specific affective thought inductions, such as a depressivefocus condition or worry-focus, may clarify the extent to which the neural responses demonstrated in this study are specific to anger.

While the present study demonstrated differences between women with BPD and healthy controls, it is not clear the extent to which these effects are specific 
to BPD. SCID-II interviews were only conducted to evaluate BPD criteria, and therefore the extent of co-morbid other disorders is not known, although their existence likely. The BPD sample endorsed elevated scores on depression and PTSD screeners; however, these values are similar to those found in other BPD clinical samples [11], and screeners best distinguish between individuals with diagnoses and healthy controls but do not function optimally within other clinical samples [59]. Both depressive and PTSD symptom endorsement were highly correlated with BPD symptoms in the present study, as to be expected in this sample of only individuals with $\mathrm{BPD}$ and healthy controls. NAcc activation during provocation-focused (vs neutral) thought was significantly associated with BPD symptoms only; however, given the small sample size and potential restriction of range issues, the specificity of these findings should still be interpreted cautiously. Given that excluding commonly comorbid diagnoses (e.g., depressive disorders) can limit the external validity of a BPD sample, extending this work with clinical comparison groups (such as individuals with depression and/or anxiety disorders) would best clarify the specificity of these findings to BPD. It is possible these findings may be attributable to comorbid psychopathology or to a broader transdiagnostic process relevant to multiple diagnoses, including BPD. This study is also limited by a small sample size; follow-up studies with larger samples could explore these theories with greater power. Using larger samples would also allow for testing of potential moderators of these effects within the BPD group, including co-morbid diagnoses and other individual differences, which is especially important given the heterogeneous nature of the diagnosis. The current study also used female participants; future work should examine these effects in men, as well as determining whether sex may moderate the effects.

\section{Conclusions}

These findings have potential clinical implications for the treatment of BPD. If provocation-focused rumination following interpersonal criticism is a rewarding experience for these women, that may explain why they do it despite the long-term negative consequences. It also may make it difficult for them to stop engaging in provocation-focused rumination or to be motivated to try to stop, even if they are aware of its detrimental effects. This rewardsensitization could also foster other addictive tendencies. Bidirectional cross-sensitization has been demonstrated between substances and naturally occurring rewards, such as food and sex [60-62], with sensitization to one stimuli increasing responses to the other due to common neural mechanisms [63]. Individuals with BPD demonstrate elevated rates of impulsive behaviors such as substance abuse, binge-eating, and risky sexual behavior [1]; early sensitization to provocation-focused rumination-related reward could contribute to these vulnerabilities.
Interventions targeting anger rumination may need to utilize techniques designed for other behaviors that are rewarding in the short term, such as substance abuse. Motivational interviewing [64], for example, may help individuals acknowledge the effects of their behavior and increase their readiness to make changes. Current approaches to BPD treatment, such as dialectical behavior therapy DBT; $[65,66]$, teach mindfulness skills for increasing awareness of thoughts and emotions and skills for managing urges and tolerating distress without engaging in risky behaviors. Applying these specifically to anger rumination may help patients to identify when they feel distress from interpersonal interactions, to recognize when they are engaging in anger rumination, and to substitute less harmful behaviors for managing those emotions. Increasing acceptance of initial emotional reactivity to criticism may also reduce the value of the reward of externalizing blame. Cognitive emotion regulation strategies have been shown to affect striatal responses to reward cues in a non-clinical sample [67]. Further research should examine whether interventions attenuate the NAcc activation found in the present study during anger rumination for individuals with BPD or whether any strategies may help with self-control despite sustained NAcc activation.

\section{Endnotes}

${ }^{1}$ The first two participants completed a longer version of this task, in which blocks consisted of 12 statements (15 s per statement; 180 s per block).

${ }^{2}$ When analyzed separately, ANOVA results with original ROI estimates all demonstrate the same patterns of significance as those with the bilateral indices created.

\begin{abstract}
Abbreviations
ACC: anterior cingulate cortex; Al: anterior insula; ARS: Anger Rumination Scale; BPD: borderline personality disorder; CES-D: Center for Epidemiological Studies Depression Scale; dACC: dorsal anterior cingulate cortex; DMPFC: dorsomedial prefrontal cortex; DRT: Directed Rumination Task; fMRI: functional magnetic resonance imaging; NAcc: nucleus accumbens; PAI-BOR: Personality Assessment Inventory-Borderline Scale; PCL: PostTraumatic Stress Disorder Checklist; PET: positron emission topography; ROI: region of interest; SCID-II: Structured Clinical Interview for the DSM-IV Axis II; VLPFC: ventrolateral prefrontal cortex
\end{abstract}

\section{Acknowledgements \\ The authors would like to thank David Powell for his technical help in the running of this study, and Paul J. Geiger and Jacob J. Folsom for their assistance with data collection. We would also like to thank Tory A. Eisenlohr-Moul for valuable feedback on this manuscript.}

\section{Funding}

This study was supported by a research support grant from the University of Kentucky. JRP is supported by a grant from the National Institute of Mental Health (T32MH019927), and ECW is supported by the National Center for Advancing Translational Sciences (KL2TR001109). The content is solely the responsibility of the authors and does not necessarily reflect the official views of the National Institutes of Health or the University of Kentucky. None of the funding bodies were involved in the design of the study, the collection, analysis, and interpretation of data, or in writing the manuscript. 


\section{Availability of data and materials}

The datasets used and/or analyzed during the current study are available from the corresponding author on reasonable request.

\section{Authors' contributions}

JRP and DSC developed the study concept. JRP, DSC, CND, and RAB contributed to the study design. Participant recruitment, psychological testing, and risk management was conducted by JRP, under the supervision of RAB. DSC conducted fMRI testing. DSC and ECW conducted fMRI data reduction, and further data analysis was conducted by JRP. JRP drafted the paper, DSC and ECW provided the FMRI-related methods and critical revisions, and ECW created the fMRI images included as figures. All authors approved the final version of the paper for submission.

\section{Ethics approval and consent to participate}

All study activities were approved by the Medical Institutional Review Board of the University of Kentucky, and informed consent was obtained from all study participants, including further consent obtained to use data following debriefing procedures.

\section{Consent for publication}

Not applicable.

\section{Competing interests}

The authors declare that they have no competing interests.

\section{Publisher's Note}

Springer Nature remains neutral with regard to jurisdictional claims in published maps and institutional affiliations.

\section{Author details}

${ }^{1}$ Department of Psychiatry and Human Behavior, Alpert Medical School of Brown University and Rhode Island Hospital, 700 Butler Drive, Providence, RI 02906, USA. ${ }^{2}$ Department of Psychology, Virginia Commonwealth University, Richmond, VA, USA. ${ }^{3}$ Department of Psychiatry, University of North Carolina at Chapel Hill School of Medicine, Chapel Hill, NC, USA. ${ }^{4}$ Department of Psychology, University of Kentucky, Lexington, KY, USA.

Received: 25 April 2017 Accepted: 4 January 2018

\section{Published online: 16 January 2018}

\section{References}

1. American Psychiatric Association. Diagnostic and statistical manual of mental disorders (DSM-5 ). Arlington, VA: American Psychiatric Pub; 2013.

2. Nolen-Hoeksema S, Wisco BE, Lyubomirsky S. Rethinking rumination. Perspect Psychol Sci. 2008;3:400-24.

3. Papageorgiou C, Wells A. Metacognitive beliefs about rumination in recurrent major depression. Cogn Behav Pract. 2001;8:160-4.

4. Watkins E, Baracaia S. Why do people ruminate in dysphoric moods? Personal Individ Differ. 2001;30:723-34.

5. Baer RA, Sauer SE. Relationships between depressive rumination, anger rumination, and borderline personality features. Personality Disorders: Theory, Research, and Treatment. 2011;2:142-50.

6. Peters JR, Eisenlohr-Moul TA, Upton BT, Talavera NA, Folsom JJ, Baer RA. Characteristics of repetitive thought associated with borderline personality features: a multimodal investigation of ruminative content and style. J Psychopathol Behav Assess. 2017;39:456-66.

7. Bushman BJ, Bonacci AM, Pedersen WC, Vasquez EA, Miller N. Chewing on it can chew you up: effects of rumination on triggered displaced aggression. J Pers Soc Psychol. 2005;88:969-83.

8. Peled M, Moretti MM. Ruminating on rumination: are rumination on anger and sadness differentially related to aggression and depressed mood? J Psychopathol Behav Assess. 2009;32:108-17.

9. Rusting CL, Nolen-Hoeksema S. Regulating responses to anger: effects of rumination and distraction on angry mood. J Pers Soc Psychol. 1998;74:790-803.

10. Gardner FL, Moore ZE. Understanding clinical anger and violence: the anger avoidance model. Behav Modif. 2008;32:897-912.

11. Rüsch N, Lieb K, Göttler I, Hermann C, Schramm E, Richter H, Jacob GA, Corrigan PW, Bohus M. Shame and implicit self-concept in women with borderline personality disorder. Am J Psychiatry. 2007;164:500-8.
12. Gratz K, Rosenthal M, Tull M, Lejuez C. An experimental investigation of emotional reactivity and delayed emotional recovery in borderline personality disorder: the role of shame. Compr Psychiatry. 2010;51:275-85.

13. Peters JR, Geiger PJ, Smart LM, Baer RA. Shame and borderline personality features: the potential mediating role of anger and anger rumination. Personality Disorders: Theory, Research, and Treatment. 2014;5:1-9.

14. Staebler K, Helbing E, Rosenbach C, Renneberg B. Rejection sensitivity and borderline personality disorder. Clinical Psychology and Psychotherapy. 2010;18:275-83.

15. Ayduk Ö, Zayas V, Downey G, Blum Cole A, Shoda Y, Mischel W. Rejection sensitivity and executive control: joint predictors of borderline personality features. J Res Pers. 2008;42:151-68.

16. Peters JR, Smart LM, Baer RA. Dysfunctional responses to emotion mediate the cross-sectional relationship between rejection sensitivity and borderline personality features. J Personal Disord. 2015:29:231-40.

17. Berenson KR, Downey G, Rafaeli E, Coifman KG, Paquin NL. The rejectionrage contingency in borderline personality disorder. J Abnorm Psychol. 2011;120:681-90.

18. Harmon-Jones C, Schmeichel BJ, Mennitt E, Harmon-Jones E. The expression of determination: similarities between anger and approach-related positive affect. J Pers Soc Psychol. 2011:100:172-81.

19. Reichenberger J, Eibl JJ, Pfaltz M, Wilhelm FH, Voderholzer U, Hillert A, Blechert J. Don"t praise me, don"t chase me: emotional reactivity to positive and negative social-evaluative videos in patients with borderline personality disorder. J Personal Disord. 2016:1-15.

20. Eisenberger NI. Does rejection hurt? An fMRI study of social exclusion. Science. 2003;302:290-2.

21. Eisenberger NI, Lieberman MD. Why rejection hurts: a common neural alarm system for physical and social pain. Trends Cogn Sci. 2004;8:294-300.

22. Domsalla M, Koppe G, Niedtfeld I, Vollstädt-Klein S, Schmahl C, Bohus M, Lis S. Cerebral processing of social rejection in patients with borderline personality disorder. Soc Cogn Affect Neurosci. 2014;9:1789-97.

23. Wager TD, Davidson ML, Hughes BL, Lindquist MA, Ochsner KN. Prefrontalsubcortical pathways mediating successful emotion regulation. Neuron. 2008;59:1037-50.

24. Kawamoto $\mathrm{T}$. Is dorsal anterior cingulate cortex activation in response to social exclusion due to expectancy violation? An fMRI study. Front Evol Neurosci. 2012:4:1-10.

25. Riva P, Romero Lauro $\sqcup$, DeWall CN, Bushman BJ. Buffer the pain away: stimulating the right ventrolateral prefrontal cortex reduces pain following social exclusion. Psychol Sci. 2012;23:1473-5

26. Riva P, Romero Lauro L, Vergallito A, DeWall CN, Bushman BJ. Electrified emotions: modulatory effects of transcranial direct stimulation on negative emotional reactions to social exclusion. Soc Neurosci. 2015;10:46-54.

27. Denson TF, Pedersen WC, Ronquillo J, Nandy AS. The angry brain: neural correlates of anger, angry rumination, and aggressive personality. J Cogn Neurosci. 2009:21:734-44.

28. Floresco SB. The nucleus accumbens: an interface between cognition, emotion, and action. Annu Rev Psychol. 2015:66:25-52.

29. Diekhof EK, Kaps L, Falkai P, Gruber O. The role of the human ventral striatum and the medial orbitofrontal cortex in the representation of reward magnitude: an activation likelihood estimation meta-analysis of neuroimaging studies of passive reward expectancy and outcome processing. Neuropsychologia. 2012;50:1252-66.

30. Kühn S, Gallinat J. The neural correlates of subjective pleasantness. Neurolmage. 2012;61:289-94.

31. Bartra O, McGuire JT, Kable JW. The valuation system: a coordinate-based meta-analysis of BOLD fMRI experiments examining neural correlates of subjective value. Neurolmage. 2013:1-19.

32. Berridge KC, Kringelbach ML. Neuroscience of affect: brain mechanisms of pleasure and displeasure. Curr Opin Neurobiol. 2013;23:294-303.

33. Costa VD, Lang PJ, Sabatinelli D, Versace F, Bradley MM. Emotional imagery: assessing pleasure and arousal in the brain's reward circuitry. Hum Brain Mapp. 2010;31:1446-57.

34. Volkow ND, Fowler JS, Wang G-J, Swanson JM. Dopamine in drug abuse and addiction: results from imaging studies and treatment implications. Mol Psychiatry. 2004;9:557-69.

35. Robinson TE, Berridge KC. The incentive sensitization theory of addiction: some current issues. Philos Trans R Soc Lond B Biol Sci. 2008;363:3137-46.

36. Stice E, Spoor S, Ng J, Zald DH. Relation of obesity to consummatory and anticipatory food reward. Physiol Behav. 2009;97:551-60. 
37. Pearson CM, Chester DS, Powell D, Wonderlich SA, Smith GT. Investigating the reinforcing value of binge anticipation. Int J Eat Disord. 2016:1-3.

38. Chester DS, DeWall CN. The pleasure of revenge: retaliatory aggression arises from a neural imbalance toward reward. Soc Cogn Affect Neurosci. 2016;11:1173-82

39. Enzi B, Doering S, Faber C, Hinrichs J, Bahmer J, Northoff G. Reduced deactivation in reward circuitry and midline structures during emotion processing in borderline personality disorder. World J Biol Psychiatry. 2013;14:45-56.

40. First MB, Gibbon M. User's guide for the structured clinical interview for Dsm-IV Axis II personality disorders. Arlington, VA: American Psychiatric Publishing, Incorporated; 1997.

41. Morey LC. Personality assessment inventory professional manual. 2nd ed. Psychological Assessment Resources Incorporated: Odessa, FL; 2007.

42. Trull TJ, Useda D, Conforti K, Doan BT. Borderline personality disorder features in nonclinical young adults: 2. Two-year outcome. 1997;106:307.

43. Sukhodolsky DG, Golub A, Cromwell EN. Development and validation of the anger rumination scale. Personal Individ Differ. 2001;31:689-700.

44. Radloff LS. The CES-D scale a self-report depression scale for research in the general population. Appl Psychol Meas. 1977;

45. Blanchard EB, Jones-Alexander J, Buckley TC, Forneris CA. Psychometric properties of the PTSD checklist (PCL). Behav Res Ther. 1996;34:669-73.

46. Bushman BJ, Baumeister RF. Threatened egotism, narcissism, self-esteem, and direct and displaced aggression: does self-love or self-hate lead to violence? J Pers Soc Psychol. 1998;75:219-29.

47. Woolrich MW, Jbabdi S, Patenaude B, Chappell M. Bayesian analysis of neuroimaging data in FSL. Neurolmage. 2009;45:S173-86.

48. Smith SM, Jenkinson M, Woolrich MW, Beckmann CF, Behrens TEJ, Johansen-Berg H, Bannister PR, De Luca M, Drobnjak I, Flitney DE, Niazy RK, Saunders J, Vickers J, Zhang Y, De Stefano N, Brady JM, Matthews PM. Advances in functional and structural MR image analysis and implementation as FSL. Neurolmage. 2004;23(Suppl 1):S208-19.

49. Jenkinson M, Bannister P, Brady M, Smith S. Improved optimization for the robust and accurate linear registration and motion correction of brain images. Neurolmage. 2002;17:825-41.

50. Smith SM. Fast robust automated brain extraction. Hum Brain Mapp. 2002 17:143-55.

51. Maldjian JA, Laurienti PJ, Kraft RA, Burdette JH. An automated method for neuroanatomic and cytoarchitectonic atlas-based interrogation of fMRI data sets. Neurolmage. 2003;19:1233-9.

52. Cohen J. Statistical power analysis for the behavioral sciences. 2 nd ed. New York: Routledge; 1988

53. Watanabe N, Sakagami M, Haruno M. Reward prediction error signal enhanced by striatum-amygdala interaction explains the acceleration of probabilistic reward learning by emotion. J Neurosci. 2013;33:4487-93.

54. Hampshire A, Chamberlain SR, Monti MM, Duncan J, Owen AM. The role of the right inferior frontal gyrus: inhibition and attentional control. Neurolmage. 2010;50:1313-9.

55. Ruocco AC, Amirthavasagam S, Choi-Kain LW, McMain SF. Neural correlates of negative emotionality in borderline personality disorder: an activationlikelihood-estimation meta-analysis. Biol Psychiatry. 2013:73:153-60.

56. Ducasse D, Courtet P, Olié E. Physical and social pains in borderline disorder and neuroanatomical correlates: a systematic review. Curr Psychiatry Rep. 2014;16:443.

57. Sauer C, Arens EA, Stopsack M, Spitzer C, Barnow S. Emotional hyperreactivity in borderline personality disorder is related to trauma and interpersonal themes. Psychiatry Res. 2014;220:468-76.

58. Limberg A, Barnow S, Freyberger HJ, Hamm AO. Emotional vulnerability in borderline personality disorder is cue specific and modulated by traumatization. Biol Psychiatry. 2011;69:574-82.

59. McDonald SD, Calhoun PS. The diagnostic accuracy of the PTSD checklist: a critical review. Clin Psychol Rev. 2010;30:976-87.

60. Fiorino DF, Phillips AG. Facilitation of sexual behavior and enhanced dopamine efflux in the nucleus accumbens of male rats after Damphetamine-induced behavioral sensitization. J Neurosci. 1999;19:456-63.

61. Avena NM, Hoebel BG. A diet promoting sugar dependency causes behavioral cross-sensitization to a low dose of amphetamine. Neuroscience. 2003;122:17-20.

62. Avena NM, Hoebel BG. Amphetamine-sensitized rats show sugar-induced hyperactivity (cross-sensitization) and sugar hyperphagia. Pharmacol Biochem Behav. 2003;74:635-9.
63. Antelman SM, Eichler AJ, Black CA, Kocan D. Interchangeability of stress and amphetamine in sensitization. Science. 1980;207:329-31.

64. Miller WR, Rollnick S. Motivational interviewing: helping people change. 3rd ed. New York: Guilford Press; 2013.

65. Linehan M. Cognitive-behavioral treatment of borderline personality disorder. New York: Guilford Press; 1993.

66. Linehan MM. DBT ${ }^{\oplus}$ skills training manual. Second Edition: Guilford Publications; 2014.

67. Delgado MR, Gillis MM, Phelps EA. Regulating the expectation of reward via cognitive strategies. Nat Neurosci. 2008;11:880-1.

\section{Submit your next manuscript to BioMed Central and we will help you at every step:}

- We accept pre-submission inquiries

- Our selector tool helps you to find the most relevant journal

- We provide round the clock customer support

- Convenient online submission

- Thorough peer review

- Inclusion in PubMed and all major indexing services

- Maximum visibility for your research

Submit your manuscript at www.biomedcentral.com/submit
Biomed Central 